\title{
Efectividad de la artroscopia temporomandibular para el diagnóstico de adherencias intrarticulares y perforaciones discales: revisión sistemática de la literatura
}

\author{
Effectiveness of Temporomandibular Arthroscopy for the Diagnosis \\ of Intra-articular Adhesions and Disc Perforations: Systematic \\ Literature Review
}

Luis Vicente González Rodríguez Odontólogo, Universidad de Carabobo, Valencia, Venezuela. Cirujano maxilofacial, Pontificia Universidad Javeriana, Bogotá, Colombia.

Liz Beth Bello Cubillos Odontóloga, Periodoncista, Pontificia Universidad Javeriana, Bogotá, Colombia.

CÓMO CITAR ESTE ARTÍ́CULO González LV, Bello LB. Efectividad de la artroscopia temporomandibular para el diagnóstico de adherencias intrarticulares y perforaciones discales: revisión sistemática de la literatura. Univ Odontol. 2015 Ene-Jun; 34(72): 47-56. http:// dx.doi.org/10.11144/Javeriana.uo34-72.eatd

doi:10.11144/Javeriana.uo34-72.eatd

Recibido para publicación: 12/11/2014 Aceptado para publicación: 15/06/2015

Disponible en: http://www.javeriana.edu.co/ universitasodontologica

\section{RESUMEN}

Antecedentes: Diferentes estudios actuales identifican la artroscopia temporomandibular como una técnica operatoria. Sin embargo, la gran utilidad diagnóstica de este método es muy poco abordada en la literatura. Asimismo, pocas investigaciones describen su precisión diagnóstica en el manejo de las alteraciones degenerativas intrarticulares. Objetivo: Determinar la eficacia de la artroscopia temporomandibular en el diagnóstico de patologías articulares frente a la resonancia magnética nuclear, por medio de una revisión sistemática de la literatura. Métodos: Se realizó una búsqueda de artículos en diferentes bases de datos. Se aplicaron los siguientes criterios de inclusión: estudios clínicos abiertos en humanos, estudios con enfoque diagnóstico que mostraran especificidad y sensibilidad de la técnica de artroscopia diagnóstica y resonancia magnética nuclear, referentes a patologías de desplazamiento anterior del disco con reducción o sin esta, adherencias del espacio articular superior y perforaciones discales, e idioma inglés. La búsqueda inicial arrojó un total de 632 títulos, de los cuales se seleccionaron 3 artículos a los cuales se les realizó análisis de calidad. Resultados: Los estudios muestran una eficacia diagnóstica del 92,39 $\%$ de perforaciones discales y del $61 \%$ para adherencias intrarticulares con resonancia magnética. El diagnóstico con artroscopia de adherencias y perforaciones discales tuvo una sensibilidad y especificidad del $97 \%$. Conclusiones: Los estudios analizados muestran una baja precisión diagnóstica con deficiente sensibilidad y especificidad de la resonancia magnética nuclear para el diagnóstico de adherencias. Por otra parte, la artroscopia presentó una especificidad y sensibilidad diagnóstica elevada. Sin embargo, no hubo diferencias significativas entre ambas técnicas.

PALABRAS CLAVE

adherencias intrarticulares; artroscopia temporomandibular; perforación discal; resonancia magnética nuclear

\section{ÁREAS TEMÁTICAS}

Articulación temporomandibular; diagnóstico; patología intrarticular degenerativa

\section{ABSTRACT}

Background: Several recent studies describe temporomandibular arthroscopy as an operatory technique. However, the diagnostic usefulness of this method has not been examined much in the literature. Likewise, a few studies describe its accuracy to diagnose intra-articular degenerative anomalies. Purpose: To determine the effectiveness of temporomandibular arthroscopy to diagnose articular pathologies, when compared to nuclear magnetic resonance, through a systematic review of literature. Methods: An article search was conducted using several databases. The inclusion criteria included: open clinical studies; in humans; diagnostic studies that show specificity and sensitivity of diagnostic arthroscopy and nuclear magnetic resonance regarding articular disc anterior displacement with or without reduction, upper joint space adherence and disc perforations; and English language. The initial search showed 632 titles, of which 3 were chosen to conduct the quality analysis. Results: Studies show $92.39 \%$ diagnostic effectiveness of magnetic resonance for disc perforations and $61 \%$ for intra-articular adherences. Diagnostics of adherences and perforations with arthroscopy had a sensitivity and specificity of $97 \%$. Conclusions: Studies analyzed show low diagnostic accuracy with low sensitivity and specificity of nuclear magnetic resonance to diagnose adherences. On the other side, arthroscopy showed high specificity and sensitivity. However, there were not statistically significant differences between both techniques.

\section{KEYWORDS}

disc perforation; intra-articular adhesions; nuclear magnetic resonance, temporomandibular arthroscopy

THEMATIC FIELDS

Degenerative intra-articular pathology; diagnostics; temporomandibular joint 


\section{INTRODUCCIÓN}

Los trastornos temporomandibulares se definen como un amplio grupo de problemas clínicos relacionados con los músculos masticatorios y la articulación temporomandibular, que involucran los componentes estructurales blandos y duros (1). Existe una prevalencia del 6-12\% de la sintomatología dolorosa articular en la población, la cual se observa con mayor frecuencia en personas con disfunción oclusal (2). Un buen diagnóstico clínico es fundamental y su importancia radica en determinar con exactitud el grado de alteración degenerativa que puedan presentar el disco articular o los tejidos sinoviales. Por tal motivo, no es confiable usar solamente el examen clínico para determinar el estado de las alteraciones articulares internas (3). La utilización de métodos imagenológicos no invasivos, como la resonancia magnética nuclear, ha mostrado ser una excelente y confiable herramienta diagnóstica en las alteraciones intrarticulares (4). Sin embargo, la eficacia diagnóstica de la resonancia magnética puede carecer de sensibilidad y especificidad en patologías degenerativas, como las adherencias intrarticulares y las perforaciones discales (5).

\section{Herramientas diagnósticas en el estudio de los trastornos temporomandibulares}

En la actualidad, el estudio de la articulación temporomandibular se debe llevar a cabo con la exploración clínica, la aplicación de técnicas imagenológicas de alta precisión y la visualización endoscópica de la articulación, para correlacionar los hallazgos y establecer un estadio clínico adecuado como diagnóstico principal. El desplazamiento discal (anterior, anteromedial o anterolateral) es uno de los hallazgos más comunes en pacientes que presentan sintomatología temporomandibular (6). La ubicación anatómica del disco en la resonancia magnética nuclear permite saber la cantidad de desplazamiento. Sin embargo, esta herramienta no permite apreciar la cantidad de inflamación y la degeneración de los tejidos sinoviales (7).

\section{Artroscopia temporomandibular}

La primera artroscopia temporomandibular en la literatura maxilofacial fue reportada por Ohnishi, en 1975, en el boletín de la Facultad de Medicina de la Universidad de Tokio (8). Su artículo presenta la metodología y los hallazgos del examen artroscópico temporomandibular. La descripción se basó en la técnica de punción, la inserción de la óptica en el espacio articular superior, así como la punción con una aguja drenaje para la continua efusión de solución salina. En 1986, el primer reporte de la técnica artroscópica fue realizado por Joseph McCain en la reunión anual de la Asociación Estadounidense de Cirugía Oral yMaxilofacial en Washington D. C. (9). Las ventajas de este procedimiento se enfocaron en la observación de los movimientos y las estructuras sinoviales internas.

Técnica de artroscopia diagnóstica

Bajo anestesia general e intubación nasotraqueal, se realiza el marcaje de las áreas de punción preauricular. Para realizar un abordaje superior posterolateral del espacio articular superior, Holmlund, Hellsing y Wredmark (10) describieron una línea que va desde el canto lateral del ojo a la porción media del trago ipsilateral. La concavidad máxima de la fosa glenoidea generalmente se encuentra $10 \mathrm{~mm}$ anterior al trago y $2 \mathrm{~mm}$ inferior a la línea trago-cantal. El procedimiento requiere una punción inicial con un trocar en el espacio articular superior. Con el pulgar de la mano no dominante se palpan el arco cigomático y la concavidad de la fosa glenoidea. Se tienen en cuenta las marcas descritas y se realiza la punción con el trocar en dirección al borde inferior del arco cigomático. Al tener contacto con el borde óseo, se realizan movimientos laterales para desinsertar las fibras de la cápsula articular y el periostio. Se procede a cambiar la inclinación y se penetra el espacio articular superior, realizando movimientos rotatorios hasta sentir la resistencia que hace la cápsula articular hasta una profundidad de 20-25 mm. Debe asegurase de no invadir la porción medial de la cápsula articular. Una vez adentro del espacio articular, se lava con solución salina y se introduce la óptica de $1,9 \mathrm{~mm}$ de diámetro y 30 grados (figura 1). Se verifica la visualización de las estructuras y se coloca una aguja de flujo de salida, ubicada $5 \mathrm{~mm}$ adelante y $5 \mathrm{~mm}$ por debajo de la punción inicial. Es importante mantener un flujo constante para evitar el colapso de la cápsula articular. Se inicia el reconocimiento de las estructuras anatómicas y la evaluación de los tejidos sinoviales. 
FIGURA 1

LÍNEA TRAGO-CANTAL DE HOLMLUND-HELLSING: A) ABORDAJE SUPERIOR POSTEROLATERAL 10 MM ANTERIOR AL TRAGO Y 2 MM INFERIOR. B) PUNTO DE AGUJA DE DRENAJE 15 MM ANTERIOR AL TRAGO Y 7 MM INFERIOR A LA LÍNEA tRago-CANTAL. C) ÓPtica de 30 GRAdos 1,9 MM DE DIÁMETRO STORz®. D) CAMISA DE TROCAR. E) TROCAR ROMO. COLOCACIÓN DE LA AGUJA DE DRENAJE DURANTE LA ARTROSCOPIA DIAGNÓSTICA

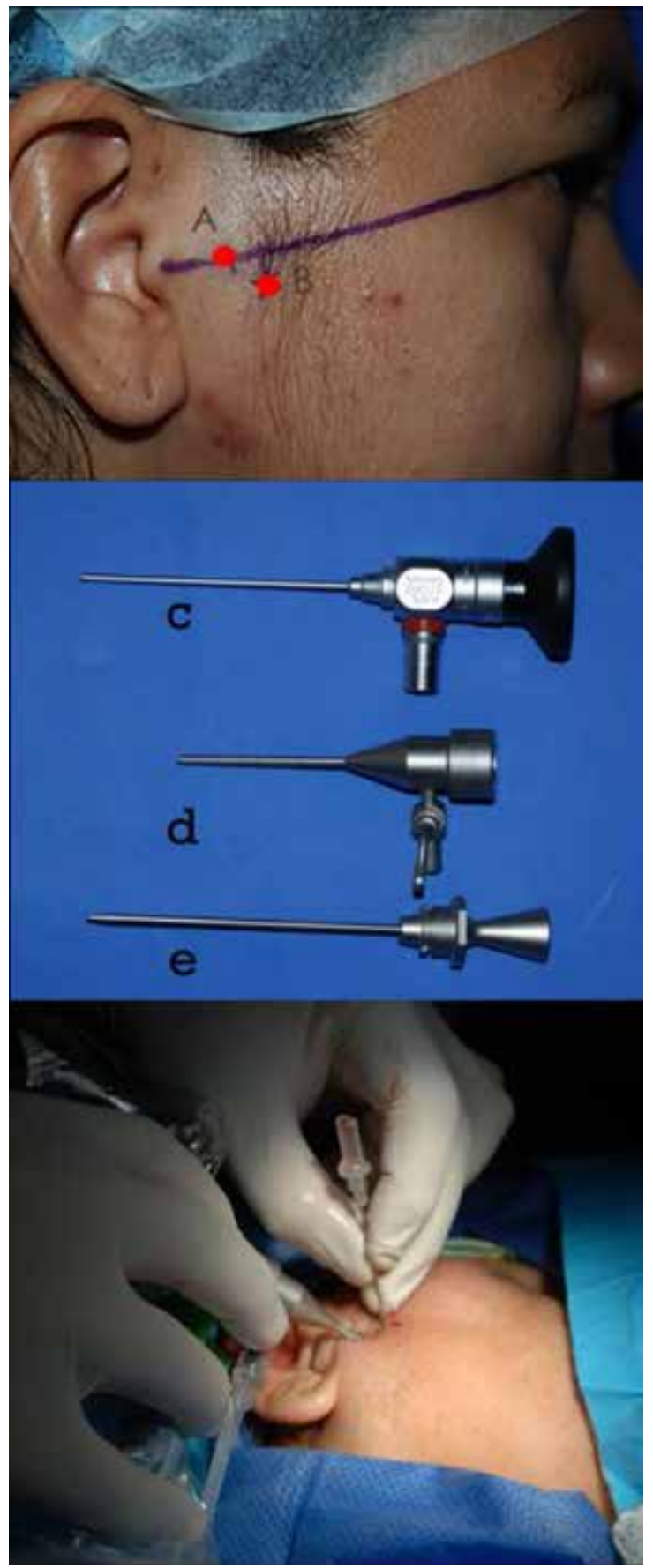

Signos artroscópicos de desplazamiento anterior del disco articular, adherencias y condromalacia

La visión directa del disco articular en el espacio articular superior permite identificar una estructura de superficie avascular y aspecto nacarado que presenta un límite posterior, el cual debe iniciar en la eminencia temporal, pues es un punto importante durante el examen artroscópico (11). El principal signo que indica desplazamiento anterior es el hecho de visualizar un cóndilo recubierto por una estructura vascularizada, como son el ligamento posterior o los tejidos retrodiscales. McCain hace referencia al término roofing, que permite verificar y cuantificar la cantidad de desplazamiento del disco (12). Esta verificación se realiza bajo el procedimiento de artroscopia en el espacio articular superior de la zona intermedia y su relación con la zona retrodiscal. Cuando la unión posterior del disco con el tejido retrodiscal se visualiza en relación con la vertiente posterior de la eminencia articular del cóndilo temporal, se dice que es un roofing del $100 \%$, lo que relacionaría un disco en posición adecuada, ya que el tejido retrodiscal no cubre el cóndilo mandibular. A medida que el disco es desplazado anteriormente, disminuye el roofing. Esto es proporcional a la gravedad del cuadro. La valoración se realiza en boca abierta y se hace hincapié en que la unión de la banda posterior y el disco estén por detrás del eje mayor de la eminencia articular. A medida que empeora el roofing, disminuye hasta un $0 \%$, cuando se evidencia un cóndilo recubierto con estructuras vascularizadas.

Blaustein y Heffez (citados por Martín-Granizo) identificaron cuatro hallazgos sugerentes de desplazamiento discal (13):

- Tejido retrodiscal remodelado, que es un tejido grisáceo o blanquecino con un número de vasos muy superficiales.

- Tejido retrodiscal remodelado topográficamente, que se relaciona con el ligamento capsular medial.

- Morfología del tejido retrodiscal rosáceo con vascularización superficial (tejido retrodiscal remodelado) por el trauma constante en función.

- En la fase de exploración dinámica, el cóndilo en contacto con el tejido retrodiscal remodelado.

Los desplazamientos discales generan alteraciones estructurales del disco intrarticular y de las superficies sinoviales. Estas progresan lentamente hasta causar lo que se conoce como fenómeno de disco anclado, que clínicamente se correlaciona con bloqueo articular. Además, existen factores que contribuyen a la 
inmovilización del disco, como las patologías fibrosas y fibroóseas. Las superficies articulares están recubiertas por membranas sinoviales que se encuentran íntimamente en deslizamiento constante durante los movimientos fisiológicos del complejo cóndilo-disco y la vertiente posterior de la eminencia articular temporal. Existen dos moléculas que ayudan al mantenimiento de la integridad de las membranas sinoviales: los fosfolípidos activos de superficie y el ácido hialurónico. Este último posee un papel predominante, ya que es un mucopolisacárido de alto peso molecular que forma una película de fluido y mantiene separadas las superficies articulares, para prevenir la fricción entre ellas y proteger los fosfolípidos activos de superficie de la degradación que ejerce la fosfolipasa A2 sobre este (14).

No obstante, cuando los hábitos parafuncionales sobrecargan las estructuras internas de la articulación (presión que se ejerce sobre los tejidos subcondrales del cóndilo mandibular contra la porción posterior de la eminencia articular del temporal), se provoca un microtrauma que genera un ciclo de hipoxia-reperfusión vascular. Dicho ciclo lleva a la liberación no enzimática de especies de radicales libres de oxígeno, como superóxido y aniones hidroxilos. Estos productos generan una reacción bioquímica que resulta en la degradación del ácido hialurónico, lo que causa una disminución marcada de la viscosidad del fluido sinovial y permite que la fosfolipasa A2 esté libremente en el fluido sinovial. En consecuencia, se degradan los fosfolípidos activos de superficie para crear un efecto adhesivo en las superficies articulares (15).

De esta forma, las superficies dejan de ser lisas y se tornan superficies pegajosas. Ello genera lo que fue descrito como el efecto de copa de succión, que progresivamente genera una respuesta cicatrizal por parte de las superficies sinoviales, de manera que los hematomas subcondrales que se van formando exponen fibrina, la cual se transforma en tejido fibroso (16).

Kaminishi y colaboradores, en 1986, correlacionaron las adherencias y los desplazamientos discales y describieron las formas como se presentan las fibrosis intrarticulares (16). Las bandas simples fibrosas son la forma más simple de fibrosis intrarticular o la más elemental forma de aparición de adherencias intrarticulares. Su apariencia oscila entre: a) una vía fibrosa que discurre entre las superficies articulares y se observa en los recesos mediales de la cápsula; b) bandas fibrosinoviales, que son iguales a las anteriores pero recubiertas de tejido sinovial (son más frecuentes en el espacio intermedio e indican una lesión crónica), y c) falsas paredes (pseudowalls), que atraviesan transversalmente la articulación de medial a lateral y se pueden observar en recesos anteriores y posteriores de la cavidad articular y se relacionan ampliamente con el fenómeno de disco anclado.

El desplazamiento anterior del disco no es un hallazgo único durante la artroscopia temporomandibular. Se debe evaluar la integridad sinovial de la vertiente posterior de la eminencia articular del temporal. Esto ayuda a diagnosticar la presencia de condromalacia. La gravedad de la condromalacia obedece al estrés y las fuerzas comprensivas que generan microtrauma e inducen lesiones del condrocito y posterior liberación de catepsinas B y metaloproteinasas, que terminan en degradación de fibras colágenas de la matriz fibrocartilaginosa y progresiva degeneración $\operatorname{articular}(17,18)$. Se reconocen cuatro tipos de condromalacia diagnosticados artroscópicamente. El tipo I es un ligero ablandamiento de la superficie articular que se evalúa con un gancho palpador que se introduce por un tercer canal de operación. En el tipo II ya se evidencian arrugas en la superficie fibrocartilaginosa por degradación de las fibras colágenas profundas. El tipo III presenta exposición del fibrocartílago con fibrilaciones de este durante el lavado. En el tipo IV ya hay una degradación profunda y se reconocen áreas de exposición del hueso subcondral, así como perforación discal (18).

\section{Indicaciones de la técnica artroscópica diag- nóstica según la Asociación Estadounidense de Cirugía Maxilofacial (1988)}

La artroscopia diagnóstica se considera un procedimiento que está principalmente indicado en aquellas condiciones articulares que requieren un examen directo. Se efectúa con el fin de confirmar la sospecha clínica y estadificar la enfermedad, principalmente cuando no puedan ser confirmados por otros medios de evaluación (19). Son casos en que la artroscopia puede ser útil:

- Dolor preauricular temporomandibular inexplicable que no responde a la terapia médica durante más de seis meses, sin mejoría o resultados exitosos.

- Confirmación de otros hallazgos diagnósticos que puedan garantizar el éxito de intervención quirúrgica (cantidad de desplazamiento discal).

- Realización de biopsias en sospecha de enfermedad y lesión, para confirmación histopatológica.

Se reconoce el potencial diagnóstico y terapéutico que tiene la artroscopia temporomandibular como proce- 
dimiento mínimamente invasivo (20). Su ventaja radica, sobre todo, en que se obtiene la visualización directa de los componentes anatómicos en tiempo real. Por tal motivo, el propósito de este artículo es analizar la eficacia diagnóstica de la artroscopia temporomandibular en el estudio de adherencias intrarticulares y perforaciones discales.

\section{MATERIALES Y MÉTODOS}

Este estudio es una revisión sistemática de la literatura. Se efectuó una búsqueda exhaustiva en diferentes bases de datos (Medline, ScienceDirect y Pubmed) de artículos referentes a la precisión diagnóstica de la artroscopia temporomandibular en el estudio de perforaciones discales y adherencias intrarticulares entre 1990 y 2014. Se utilizaron las siguientes palabras clave en inglés: artroscopia temporomandibular, perforaciones discales, adherencias intrarticulares, resonancia nuclear magnética y precisión diagnóstica. Se obtuvieron 632 artículos con las siguientes combinaciones de palabras:

- Temporomandibular arthroscopy AND diagnosis disc perforation AND adhesion (2 artículos, de los cuales no se escogió ninguno).

- Temporomandibular arthroscopy AND temporomandibular disorder diagnosis (539 artículos; se escogieron 2 artículos).

- MRI AND temporomandibular disorder diagnosis (80 artículos; se escogió 1 artículo).

- MRI AND temporomandibular disorder accurate diagnosis (11 artículos; se escogió 1 artículo).

Los criterios de inclusión para la selección fueron: estudios clínicos abiertos de corte transversal, en humanos, estudios con enfoque diagnóstico que demostraran especificidad y sensibilidad de la técnica de artroscopia y la resonancia magnética nuclear, población adulta, promedio de edad de 30 años, estudios con recolección de información durante más de 6 meses en centros médicos, pacientes con patologías de desplazamiento anterior del disco con reducción o sin esta, adherencias del espacio articular superior y perforaciones discales, estudios en idioma inglés. Los criterios de exclusión fueron: artículos de reporte de casos, comunicaciones cortas, estudios diagnósticos que no utilizaran medición de la especificidad y la sensibilidad de la técnica, pacientes con patologías tumorales o infecciosas temporomandibulares, estudios con resultados post mortem.
Con estos parámetros se realizó una lectura inicial de artículos por título y resumen. Teniendo una selección exacta de artículos, se procedió a una segunda revisión detallada mediante la lectura completa de estos. Los factores que se registraron con el fin de evaluar la heterogeneidad de los resultados entre los estudios fueron los siguientes:

- Diseño del estudio y periodo de evaluación.

- Número de pacientes, sitios de extracción, tipo de intervención y razón.

- Método de evaluación.

- Idoneidad del análisis estadístico.

- Sesgo de publicación.

Para la evaluación de la calidad de los artículos elegidos se tuvieron en cuenta los criterios según las guías de evaluación de literatura médica (21). Posterior al análisis, se seleccionaron tres ensayos clínicos de corte transversal: Zhang y colaboradores (22), Shen y colaboradores (23), Tzanidakis y Sidebottom (24) (tabla 1)

\section{RESULTADOS}

Se obtuvieron tres artículos de ensayo clínicos de corte transversal retrospectivo, con control de variables en la extracción de la muestra de manera aleatorizada. El estudio de Zhang y colaboradores (22) utilizó los criterios imagenológicos para adherencias intrarticulares de Rao, Farole y Karasick (25). Los investigadores evaluaron las resonancias magnéticas nucleares de sus pacientes con secuencias ponderadas en T1 y T2. Tomaron como imágenes "positivas" aquellas que presentaran discontinuidad del disco en señal baja de T1 y señal alta en fluido sinovial en T2. Identificaron como "sospechosas" las que presentaran solo señal alta en T2 de líquido sinovial y se consideraron "negativas" las que no cumplían con los criterios mencionados. El segundo estudio, de Shen y colaboradores (23), evaluó imágenes de resonancia magnética de pacientes por medio de los criterios imagenológicos de Kuribayashi y colaboradores (26). Consideraron una imagen "positiva" aquella que en ponderaciones T1 mostrara una superficie irregular y osteofitos conectados con la cortical de la fosa glenoidea que protruyeran el espacio articular superior. También incluían una señal alta en ponderaciones T2 en el área medial del disco articular. Se definieron como "sospechosas" cuando la superficie del cóndilo era irregular y presentaba osteofitos sin conexión con la cortical de la 
TABLA 1

ESTUdIOS SELECCIONADOS

\begin{tabular}{|c|c|c|c|c|c|c|}
\hline $\begin{array}{l}\text { Autor/ } \\
\text { año }\end{array}$ & $\begin{array}{c}\text { Dise- } \\
\text { ño y } \\
\text { tiempo }\end{array}$ & Muestra & $\begin{array}{c}\text { Signos clínicos/ } \\
\text { síntomas }\end{array}$ & $\begin{array}{c}\text { Estudios } \\
\text { diagnósticos }\end{array}$ & $\begin{array}{c}\text { Examinadores } \\
\text { de resonancia } \\
\text { nuclear o } \\
\text { artroscopia }\end{array}$ & $\begin{array}{c}\text { Análisis } \\
\text { estadístico }\end{array}$ \\
\hline $\begin{array}{l}\text { Zhang y } \\
\text { colabo- } \\
\text { radores, } \\
2009(22)\end{array}$ & $\begin{array}{l}\text { Ensayo } \\
\text { clínico a } \\
9 \text { meses }\end{array}$ & $\begin{array}{l}27 \text { pacientes } \\
\text { ( } 8 \text { hombres, } \\
19 \text { mujeres), } \\
\text { media de } 35 \\
\text { años, rango } \\
\text { de edad } \\
17-51\end{array}$ & $\begin{array}{l}\text { Historia de dolor } \\
\text { intrarticular, rango } \\
\text { DII } 35 \text { mm, ruido } \\
\text { articular. Evolución } \\
\text { clínica antes de la } \\
\text { artroscopia de } 17 \\
\text { meses (rango de } 2 \\
\text { meses a } 9 \text { años) }\end{array}$ & $\begin{array}{l}\text { Resonancia magné- } \\
\text { tica en secuencia T1 } \\
\text { y T2 boca abierta y } \\
\text { boca cerrada, previa } \\
\text { a exploración ar- } \\
\text { troscópica o cirugía } \\
\text { abierta }\end{array}$ & $\begin{array}{l}\text { Interpretación } \\
\text { método ciego por } \\
\text { dos examinadores. } \\
\text { Tercer examinador } \\
\text { cuando existan dife- } \\
\text { rencias diagnósticas }\end{array}$ & $\begin{array}{l}\text { Datos analizados } \\
\text { en un diagrama de } \\
\text { especificidad y sen- } \\
\text { sibilidad en el área } \\
\text { de la curva de ROC }\end{array}$ \\
\hline $\begin{array}{l}\text { Shen y } \\
\text { colabo- } \\
\text { radores, } \\
2014(23)\end{array}$ & $\begin{array}{l}\text { Ensayo } \\
\text { clínico } \\
\text { a } 83 \\
\text { meses }\end{array}$ & $\begin{array}{l}1845 \text { pa- }^{-} \\
\text {cientes ( } 231 \\
\text { hombres, } \\
1614 \text { muje- } \\
\text { res), media } \\
\text { de } 37,6 \pm \\
13,5 \text { años }\end{array}$ & $\begin{array}{l}\text { Ruido articular, } \\
\text { inflamación pre au- } \\
\text { ricular, DII < } 35 \mathrm{~mm} \text {. } \\
\text { Evolución clínica } \\
18,08 \pm 17,03 \text { meses } \\
\text { (rango de } 5 \text { meses a } \\
5 \text { años). Diagnóstico } \\
\text { de estadio III y IV de } \\
\text { Wilkes }\end{array}$ & $\begin{array}{l}\text { Resonancia magné- } \\
\text { tica en secuencia T1 } \\
\text { y T2 boca abierta y } \\
\text { boca cerrada, previa } \\
\text { a exploración ar- } \\
\text { troscópica o cirugía } \\
\text { abierta }\end{array}$ & $\begin{array}{l}\text { Interpretación mé- } \\
\text { todo ciego por dos } \\
\text { examinadores, un } \\
\text { cirujano artroscopia } \\
\text { y un radiólogo con } \\
\text { experiencia en diag- } \\
\text { nóstico de patología } \\
\text { articular. Tercer } \\
\text { examinador cuando } \\
\text { existan diferencias } \\
\text { diagnósticas }\end{array}$ & $\begin{array}{l}\text { Datos analizados } \\
\text { en un diagrama de } \\
\text { especificidad y sen- } \\
\text { sibilidad en el área } \\
\text { de la curva de ROC }\end{array}$ \\
\hline $\begin{array}{l}\text { Tzanidakis } \\
\text { y Side- } \\
\text { bottom, } \\
2013(24)\end{array}$ & $\begin{array}{l}\text { Ensayo } \\
\text { clínico a } \\
7 \text { años }\end{array}$ & 31 pacientes & $\begin{array}{l}\text { Pacientes con } \\
\text { diagnóstico estadio } \\
\text { Wilkes II, III, IV y V. } \\
\text { Todos con artrosco- } \\
\text { pia inicial fallida que } \\
\text { requerían proce- } \\
\text { dimiento abierto } \\
\text { por persistencia de } \\
\text { dolor articular }\end{array}$ & $\begin{array}{l}\text { Artroscopia diag- } \\
\text { nóstica y radiogra- } \\
\text { fías panorámicas } \\
\text { para descartar sin- } \\
\text { tomatología dental }\end{array}$ & $\begin{array}{l}\text { El mismo ciruja- } \\
\text { no que realizó la } \\
\text { artroscopia, realizó } \\
\text { el procedimiento a } \\
\text { método abierto }\end{array}$ & $\begin{array}{l}\text { Datos analizados } \\
\text { con prueba de } \text { chi }^{2} \text {, } \\
\text { probabilidades }< \\
0,05 \text { fueron consi- } \\
\text { deradas significa- } \\
\text { tivas }\end{array}$ \\
\hline
\end{tabular}

fosa glenoidea. Las imágenes eran "negativas" cuando ninguno de los criterios nombrados se observaron en las imágenes. El tercer estudio, de Tzanidakis y Sidebottom (24), presenta una relación entre artroscopia y cirugía abierta en pacientes que tuvieron tratamientos de artroscopia fallidos inicialmente y correlacionaron los hallazgos en las cirugías abiertas.

La correlación de los diagnósticos por resonancia magnética nuclear y la confirmación por artroscopia o cirugía abierta de adherencias interarticulares presenta 4 de 11 falsos positivos (tabla 2). El rango diagnóstico fue de un $36 \%$ y la eficacia diagnóstica de la resonancia magnética nuclear para adherencias fue de 55-46 \% (tabla 3). Por otro lado, los resultados de la muestra que correlacionan los diagnósticos por resonancia magnética nuclear y la confirmación por artroscopia o cirugía abierta de perforaciones discales presentó 102 verdaderos positivos de 189 articulaciones positivas, 42 verdaderos positivos en 197 articulaciones sospechosas y 2075 verdaderos negativos entre 2138 articulaciones negativas (tabla 2). La eficacia diagnóstica fue del $92,39 \%$ un área bajo la curva de ROC de 0,808 $(0,77,0,85, p<0,05)$ (tabla 3).

Los resultados del grupo de pacientes evaluados con artroscopias inicialmente y cirugías abiertas de retratamiento, por fallo de la terapéutico de la artroscopia, confirmaron los hallazgos iniciales: la artroscopia diagnosticó 17 verdaderos positivos de 17 casos, 13 perforaciones discales verdaderas positivas de $13 \mathrm{ca}$ - 
TABLA 2

DISTRIBUCIÓN DE PACIENTES MEDIANTE RELACIÓN DE RESONANCIA MAGNÉTICA Y ARTROSCÓPICA EN EL DIAGNÓSTICO DE ADHERENCIAS Y PERFORACIONES

\begin{tabular}{|c|c|c|c|c|c|}
\hline \multirow{2}{*}{ Autor } & \multirow{2}{*}{ Artroscopia o cirugía Abierta } & \multicolumn{3}{|c|}{ Resonancia magnética nuclear } & \multirow{2}{*}{ Total } \\
\hline & & Positivo & Sospechoso & Negativo & \\
\hline \multirow{3}{*}{$\begin{array}{l}\text { Zhang y } \\
\text { colaboradores, } \\
2009\end{array}$} & Verdaderos positivos para adherencias & 7 & 3 & 8 & 18 \\
\hline & Falsos positivos para adherencias & 4 & 1 & 10 & 15 \\
\hline & Total & 11 & 4 & 18 & 33 \\
\hline \multirow{3}{*}{$\begin{array}{l}\text { Shen y } \\
\text { colaboradores, } \\
2014\end{array}$} & Verdaderos positivos para perforaciones discales & 102 & 42 & 63 & 207 \\
\hline & Falsos positivos para perforaciones discales & 87 & 155 & 2075 & 2317 \\
\hline & Total & 189 & 197 & 2138 & 2524 \\
\hline
\end{tabular}

TABLA 3

ESPECIIICIDAD, SENSIBILIDAD Y EfICACIA DIAGNÓSTICA DEL DIAGNÓSTICO DE ADHERENCIAS Y PERFORACIONES POR RESONANCIA MAGNÉTICA

\begin{tabular}{ccrrr}
\hline \multicolumn{1}{c}{ Autor } & Punto de corte de la curva de ROC & Sensibilidad & Especificidad & Eficacia diagnóstica \\
\hline & $<1$ (adherencias) & $0 / 20$ & $15 / 15$ & $15 / 33(46 \%)$ \\
\multirow{2}{*}{ Zhang y colaboradores, 2009} & $\geq 1$ (adherencias) & $7 / 18$ & $11 / 15$ & $18 / 33(55 \%)$ \\
& $\geq 2$ (adherencias) & $10 / 18$ & $10 / 15$ & $20 / 33(61 \%)$ \\
& $\geq 3$ (adherencias) & $18 / 18$ & $0 / 15$ & $18 / 33(55 \%)$ \\
\cline { 2 - 4 } Shen y colaboradores, 2014 & $<1$ (perforaciones) & $0 / 207$ & $2317 / 2317$ & $2317 / 2524(91,80 \%)$ \\
& $\geq 1$ (perforaciones) & $102 / 207$ & $2230 / 2317$ & $2332 / 2524(92,39 \%)$ \\
& $\geq 2$ (perforaciones) & $144 / 207$ & $2075 / 2317$ & $2219 / 2524(87,92 \%)$ \\
& $\geq 3$ (perforaciones) & $207 / 207$ & $0 / 2317$ & $207 / 2524(8,20 \%)$ \\
\hline
\end{tabular}

TABLA 4

ESPECIFICIDAD, SENSIBILIDAD Y EFICACIA DIAGNÓSTICA DE LAS ADHERENCIAS Y PERFORACIONES POR ARTROSCOPIA DIAGNÓSTICA Y CIRUGÍA ABIERTA, DESCRITOS POR TZANIDAKIS Y SIDEBOTTOM (24)

\begin{tabular}{lccccccc}
\hline \multicolumn{1}{c}{ Hallazgos } & Artroscopia & $\begin{array}{c}\text { Cirugía } \\
\text { abierta }\end{array}$ & $\begin{array}{c}\text { Falsos } \\
\text { positivos }\end{array}$ & $\begin{array}{c}\text { Falsos } \\
\text { negativos }\end{array}$ & $\begin{array}{c}\text { Sensibilidad } \\
\text { (\%) }\end{array}$ & $\begin{array}{c}\text { Especificidad } \\
\text { (\%) }\end{array}$ & Valor p \\
\hline Adherencias & 17 & 17 & 0 & 0 & 100 & 100 & $<0,05$ \\
Cambios articulares & 3 & 3 & 0 & 0 & 100 & 100 & $<0,05$ \\
Osteofitos & 8 & 14 & 0 & 6 & 57 & 100 & $<0,05$ \\
Perforación & 13 & 13 & 1 & 1 & 93 & 95 & $<0,05$ \\
Otros & 41 & 47 & 1 & 7 & 87 & 99 & $<0,05$ \\
\hline
\end{tabular}

sos. Tuvo una especificidad y una sensibilidad del 100 \% con una eficacia diagnóstica del $97 \%$ (tabla 4).

\section{DISCUSIÓN}

La resonancia magnética nuclear se viene usando desde hace más de dos décadas como una técnica no invasiva para el diagnóstico de las alteraciones articulares. Sin embargo, estudios como el de Santler y colaboradores (27) describen la baja eficacia en la detección de adherencias intrarticulares con la resonancia magnética convencional. La técnica ar- troscópica ha revelado ser una herramienta diagnóstica importante, desde la descripción clínica de los estadios de Wilkes, que correlacionaron los hallazgos radiográficos y clínicos de un paciente, hasta que luego fueron correlacionados con hallazgos artroscópicos y constituyeron así los estadios de Wilkes (28) y Bronstein y Merrill (29) (tabla 5). 
TABLA 5

CORRELACIÓN DE HALLAZGOS CLÍNICOS/RADIOLÓGICOS CON HALLAZGOS ARTROSCÓPICOS WILKES Y BRONSTEIN

\begin{tabular}{|c|c|c|c|}
\hline Estadio & Signos/Síntomas & $\begin{array}{c}\text { Resonancia nuclear } \\
\text { magnética }\end{array}$ & Artroscopia \\
\hline Estadio I. Inicial & Clic recíproco, indoloro & $\begin{array}{l}\text { Desplazamiento discal, con } \\
\text { reducción }\end{array}$ & $\begin{array}{l}\text { Roofing del } 80 \% \text { en boca cerrada y } \\
\text { del } 100 \% \text { en boca abierta. } \\
\text { Elongación incipiente de la zona } \\
\text { bilaminar. Sinovial y resto normal }\end{array}$ \\
\hline $\begin{array}{l}\text { Estadio II. } \\
\text { Inicial-intermedio }\end{array}$ & $\begin{array}{l}\text { Chasquido más intenso y tardío } \\
\text { en la apertura, bloqueos y } \\
\text { algias ocasionales }\end{array}$ & $\begin{array}{l}\text { Desplazamiento con } \\
\text { reducción, engrosamiento } \\
\text { de la banda posterior }\end{array}$ & $\begin{array}{l}\text { Roofing del } 50 \% \text { en boca cerrada y } \\
\text { del } 100 \% \text { en boca abierta. Elongación } \\
\text { de la zona bilaminar. Sinovitis adhesiva }\end{array}$ \\
\hline $\begin{array}{l}\text { Estadio III. } \\
\text { Intermedio }\end{array}$ & $\begin{array}{l}\text { Mayor número de episodios de } \\
\text { bloqueo, dolor más intenso y } \\
\text { frecuente }\end{array}$ & $\begin{array}{l}\text { Deformidad articular } \\
\text { evidente }\end{array}$ & $\begin{array}{l}\text { Roofing del } 5 \% \text { en boca cerrada y } \\
\text { del } 15 \% \text { en boca abierta. Elongación } \\
\text { importante de la zona bilaminar. } \\
\text { Condromalacia de grados I, II o } \\
\text { III. Adherencias visibles. Sinovitis } \\
\text { prominente }\end{array}$ \\
\hline $\begin{array}{l}\text { Estadio IV. } \\
\text { Intermedio-avanzado }\end{array}$ & $\begin{array}{l}\text { Dolor crónico variable, } \\
\text { cefaleas, limitación del } \\
\text { movimiento en fases irregulares }\end{array}$ & $\begin{array}{l}\text { RMN y TAC: evidencia } \\
\text { de cambios óseos leves } \\
\text { moderados }\end{array}$ & $\begin{array}{l}\text { Hialinización o metaplasia de la banda } \\
\text { posterior. Condromalacia de grados } \\
\text { III-IV }\end{array}$ \\
\hline $\begin{array}{l}\text { Estadio V. } \\
\text { Avanzado }\end{array}$ & $\begin{array}{l}\text { Empeoramiento progresivo } \\
\text { de la función, crepitantes } \\
\text { articulares }\end{array}$ & $\begin{array}{l}\text { Gran deformidad } \\
\text { anatómica del disco. } \\
\text { TAC: signos degenerativos, } \\
\text { esclerosis subcondral, } \\
\text { geodas, osteofitos }\end{array}$ & $\begin{array}{l}\text { Fibrilación o perforación meniscal. } \\
\text { Condromalacia grado IV. Adherencias } \\
\text { fibrosas generalizadas y falsas paredes. } \\
\text { Sinovitis avanzada }\end{array}$ \\
\hline
\end{tabular}

Por otra parte, Israel y colaboradores (30) determinaron la relación entre el dolor articular, la limitación de la apertura bucal y las adherencias intrarticulares diagnosticadas artroscópicamente. Realizaron artroscopias a 126 articulaciones de 80 pacientes con sintomatología dolorosa y limitación de la apertura bucal. Los resultados de este estudio mostraron que $14 \%$ de las artroscopias no presentaron sinovitis ni adherencias, $26 \%$ presentaron sinovitis sin adherencias, $10 \%$ evidenciaron adherencias sin sinovitis y $49 \%$ mostraron adherencias y sinovitis.

Muchos estudios relacionan la clínica del paciente con alteraciones degenerativas intrarticulares mediante el diagnóstico artroscópico. Sin embargo, el conocimiento de la eficacia diagnóstica de esta técnica mínimamente invasiva no es clara y son pocos los estudios que la determinan. Venetis y colaboradores (31) realizaron la validación de la resonancia magnética artrográfica, como técnica eficaz en el diagnóstico de adherencias intrarticulares, al compararla con la artroscopia temporomandibular. Los resultados mostraron que el diagnóstico de adherencias fue del $35 \%$ y $40 \%$ en perforaciones discales. Por lo tanto, demuestra que la resonancia magnética artrográfica es una técnica en desarrollo mínimamente invasiva, ya que se debe infiltrar el espacio articular superior. Además, no existen muchas publicaciones que muestren que tiene una eficacia superior con respecto a la artroscopia en el diagnóstico de estas alteraciones intrarticulares.

En otro estudio, Zhang y colaboradores (32) realizan un análisis retrospectivo de 1822 articulaciones con desplazamientos anteriores del disco sin reducción y con. Llevaron a cabo los procedimientos artroscópicos y determinaron que el $28,76 \%$ de las articulaciones (524 de 1822 articulaciones) presentaron diagnóstico de estadio II de Wilkes sin observación de adherencias; el 25,47 \% articulaciones con estadio III de Wilkes presentaron adherencias; el 37,99 \% de las articulaciones con estadio IV de Wilkes presentaron adherencias, y el 40,37 \% de las articulaciones con diagnóstico $\mathrm{V}$ presentaron adherencias y perforaciones discales. La artroscopia muestra tener una gran facilidad para diagnosticar este tipo de patologías intrarticulares. Las adherencias intrarticulares parecen ser un hallazgo común de artroscopia temporomandibular en pacientes con estadios intermedios y avanzados de Wilkes. La eficacia diagnóstica de esta técnica ha mostrado ser elevada para la visualización de estas alteraciones. Sin embargo, las perforaciones discales 
pueden ser más complejas de diagnosticar en estadios avanzados de la enfermedad articular. Kurita y colaboradores (33) presentaron inicialmente estudios en cadáveres para determinar la eficacia diagnóstica de la artroscopia temporomandibular. Sus resultados fueron alentadores para la técnica artroscópica, ya que en 28 articulaciones detectaron un $36 \%$ de perforaciones y adherencias intrarticulares. Lo relevante de esa investigación que resaltan los autores fue la ausencia de falsos positivos.

\section{CONCLUSIONES}

Se encontró poca literatura en las revisiones de las bases de datos consultadas. Los artículos que estuvieran dirigidos a evaluar la artroscopia temporomandibular como herramienta diagnóstica eficaz tenían débil apoyo en evidencia científica. Los estudios entre 1990 y 1995 proponen la artrografía con medio de contraste y evaluación por resonancia magnética nuclear como una opción diagnóstica eficaz. Una serie de estudios actuales realza la eficacia de la artroscopia temporomandibular diagnóstica, aunque otro grupo más grande de estudios se enfoca más en la técnica como una opción quirúrgica mínimamente invasiva. Se logró encontrar ensayos clínicos aleatorizados de la artroscopia temporomandibular operativa comparada con el método abierto quirúrgico y el manejo conservador. No obstante, se hallaron y compararon estudios representativos que tomaban la artroscopia como control y punto de referencia diagnóstica, con respecto a la resonancia magnética nuclear, para determinar la eficacia diagnóstica de esta última.

Según la literatura consultada, la resonancia magnética continúa siendo la herramienta de primera opción en el estudio de las alteraciones intrarticulares. Ello se debe a que es una técnica no invasiva que muestra tener una eficacia diagnóstica elevada. Su desventaja es ser dependiente de la experiencia del evaluador. En la presente revisión bibliográfica se encontró que la eficacia diagnóstica de esta técnica es del 92,39 \%. Por otro lado, la artroscopia temporomandibular es reconocida como una técnica que presenta una gran dificultad operativa, pero posee una eficacia diagnóstica elevada para adherencias y perforaciones discales. Se encontraron estudios que respaldan una eficacia diagnóstica del $97 \%$.

Se halló que tanto la resonancia magnética nuclear como la artroscopia temporomandibular son herramientas con un nivel elevado de especificidad y sensibilidad diagnóstica y que idealmente deben ser utilizadas para apoyar el estudio y manejo de las patologías intrarticulares.

\section{RECOMENDACIONES}

En la práctica clínica es importante evaluar la posibilidad de utilizar estas técnicas diagnósticas, no limitar el estudio de la patología intrarticular a solo imágenes no invasivas. Se recomienda iniciar líneas de investigación en artroscopia temporomandibular referentes al estudio de los tejidos en tiempo real con el empleo de biopsias sinoviales y muestras de líquido sinovial para lograr la detección temprana de moléculas y marcadores tumorales que puedan diagnosticar y predecir la evolución de la patología degenerativa artrítica temporomandibular.

\section{REFERENCIAS}

1. Liu F, Steinkeler A. Epidemiology, diagnosis, and treatment of temporomandibular disorders. Dent Clin North Am. 2013 Jul; 57(3): 465-79.

2. Tucker M, Farrel B, Farrel B. Tratamiento de los trastornos temporomandibulares. En Hupp J, Ellis E, Tuker M, editores. Cirugía oral y maxilofacial contemporánea. $5 a$ ed. Madrid: Elsevier; 2010.

3. Paesani DI, Westesson PL, Hatala MP, Tallents RH, Brooks SL Accuracy of clinical diagnosis for TMJ internal derangement and arthrosis. Oral Surg Oral Med Oral Pathol. 1992 Mar; 73(3): 360-3.

4. Ahmad M, Hollender L, Anderson Q, Kartha K, Ohrbach R, Truelove EL, John MT, Schiffman EL. Research diagnostic criteria for temporomandibular disorders (RDC/TMD): development of image analysis criteria and examiner reliability for image analysis. Oral Surg Oral Med Oral Pathol Oral Radiol Endod. 2009 Jun; 107(6): 844-60.

5. Segami N, Suzuki T, Sato J, Miyamaru M, Nishimura M, Yoshimura $\mathrm{H}$. Does joint effusion on T2 magnetic resonance images reflect synovitis? Part 3. Comparison of histologic findings of arthroscopically obtained synovium in internal derangements of the temporomandibular joint. Oral Surg Oral Med Oral Pathol Oral Radiol Endod. 2003; 95: 761-6.

6. Westesson P. Imaging of the temporomandibular joint. En Thomas M, Bronstein SL, editores. Arthroscopy of the temporomandibular joint. 1a ed. Philadelphia: Saunders; 1991.

7. Aiken A, Bouloux G, Hudgins P. MR imaging of the temporomandibular joint. Magn Reson Imaging Clin N Am. 2012 Aug; 20(3): 397-412.

8. Sidney L. Development of temporomandibular joint arthroscopy. En Thomas M, Bronstein SL, editores. Arthroscopy of the temporomandibular joint. 1a ed. Philadelphia: Saunders; 1991.

9. McCain J. Arthroscopy. J Oral Maxillofac Surg. 2009 Sep; 67(9): 15-6. 
10. Holmlund A, Hellsing G, Wredmark T. Arthroscopy of the temporomandibular joint. A clinical study. Int J Oral Maxillofac Surg. 1986 Dec; 15(6): 715-21.

11. Monges Gil F. Artroscopia diagnóstica de la articulación temporomandibular. En López R, editor. Diagnóstico y tratamiento de la patología de la articulación temporomandibular. 1a ed. Madrid: Ripano; 2009.

12. González-García R, Gil-Díez Usandizaga JL, RodríguezCampo FJ. Arthroscopic anatomy and lysis and lavage of the temporomandibular joint. Atlas Oral Maxillofac Surg Clin North Am. 2011 Sep; 19(2): 131-44.

13. Martín-Granizo R. Hallazgos artroscópicos en un caso de disco articular adherido de la ATM. Rev Esp Cir Oral Maxilofac. 2005 Nov; 27(6): 344-50.

14. Nitzan DW. Arthrocentesis--incentives for using this minimally invasive approach for temporomandibular disorders. Oral Maxillofac Surg Clin North Am. 2006 Aug; 18(3): 311-28.

15. Tomida M, Ishimaru J, Miyamoto K, Mizui T, Esaki Y, Hayashi T, Murayama K, Era S, Shibata T. Biochemical aspects of the patogénesis of temporomandibular joint disorders. Asian J Oral Maxilofac Surg. 2003 Jun; 15(2): 118-27.

16. Kaminishi R, Davis C. Intracapsular fibrosis of the superior compartment of the temporomandibular joint. En Thomas M, Bronstein SL, editores. Arthroscopy of the temporomandibular joint. 1a ed. Philadelphia: Saunders; 1991.

17. Mukarami K, Segami N. Intraarticular adhesions of the temporomandibular joint. En: Clark GT, Sanders B, Bertolami $\mathrm{CN}$, editores. Advance in diagnostic and surgical arthroscopy of the temporomandibular joint. 1a ed. Philadelphia: Saunders; 1993.

18. Quinn J. Chondromalacia: pain mediator identification and significance in TMJ arthralgia. En Bell W, editor. Modern practice in orthognathic and reconstructive surgery. 9a ed. Philadelphia: Saunders; 1992.

19. Jiang Q, Qiu YT, Chen MJ, Zhang ZY, Yang C. Synovial TGF-? 1 and MMP-3 levels and their correlation with the progression of temporomandibular joint osteoarthritis combined with disc displacement: A preliminary study. Biomed Rep. 2013 Mar; 1(2): 218-22.

20. Bronstein SL. Diagnostic and operative arthroscopy. Historical perspectives and indications. Oral Maxillofac Clin North Am. 1989 Sep; 1(1): 59-68.

21. Jaeschke R, Guyatt G, Sackett DL Users' guides to the medical literature. III. How to use an article about a diagnostic test. A. Are the results of the study valid? Evidence-Based Medicine Working Group. J Am Med Assoc. 1994 Feb 2; 271(5): 389-91.

22. Zhang S, Yang C, Chen M, Fan X, Yun B, Peng Y, Yuan D. Magnetic resonance imaging in the diagnosis of intraarticular adhesions of the temporomandibular joint. $\mathrm{Br}$ J Oral Maxillofac Surg. 2009 Jul; 47(5): 389-92.

23. Shen P, Huo L, Zhang SY, Yang C, Cai XY, Liu XM. Magnetic resonance imaging applied to the diagnosis of perforation of the temporomandibular joint. J Craniomaxillofac Surg. 2014 Sep; 42(6): 874-8.

24. Tzanidakis K, Sidebottom AJ. How accurate is arthroscopy of the temporomandibular joint? A comparison of findings in patients who had open operations after arthroscopic management failed. $\mathrm{Br} \mathrm{J}$ Oral Maxillofac Surg. 2013 Dec; 51(8): 968-70.

25. Rao VM, Farole A, Karasick D. Temporomandibular joint dysfunction: correlation of MR imaging, arthrography, and arthroscopy. Radiology. 1990 Mar; 174(3 Pt 1): 6637.

26. Kuribayashi A, Okochi K, Kobayashi K, Kurabayashi T. MRI findings of temporomandibular joints with disk perforation. Oral Surg Oral Med Oral Pathol Oral Radiol Endod. 2008 Sep; 106(3): 419-25.

27. Santler G, Kärcher H, Simbrunner J. MR imaging of the TMJ. MR diagnosis and intraoperative findings. J Craniomaxillofac Surg. 1993 Oct; 21(7): 284-8.

28. Wilkes $\mathrm{CH}$. Internal derangements of the temporomandibular joint. Arch Otolaryngol Head Neck Surg 1989; 115: 469.

29. Bronstein SL, Merrill RG. Clinical staging for TMJ internal derangement: Application to arthroscopy. J Craniomandib Disord Facial Oral Pain. 1992; 6(1): 7-16.

30. Israel HA, Langevin CJ, Singer MD, Behrman DA. The relationship between temporomandibular joint synovitis and adhesions: pathogenic mechanisms and clinical implications for surgical management. J Oral Maxillofac Surg. 2006 Jul; 64(7): 1066-74.

31. Venetis G, Pilavaki M, Triantafyllidou K, Papachristodoulou A, Lazaridis N, Palladas P. The value of magnetic resonance arthrography of the temporomandibular joint in imaging disc adhesions and perforations. Dentomaxillofac Radiol. 2011 Feb; 40(2): 84-90.

32. Zhang S, Liu X, Yang C, Cai X, Chen M, Haddad MS, Yun $B$, Chen Z. Intra-articular adhesions of the temporomandibular joint: Relation between arthroscopic findings and clinical symptoms. BMC Musculoskelet Disord. 2009 Jun; 17(10): 1-6.

33. Kurita K, Bronstein SL, Westesson PL, Sternby NH. Arthroscopic diagnosis of perforation and adhesions of the temporomandibular joint: correlation with postmortem morphology. Oral Surg Oral Med Oral Pathol. 1989 Aug; 68(2): 130-4.

\section{CORRESPONDENCIA}

\author{
Luis Vicente González Rodríguez \\ luiscirujano@gmail.com
}

Liz Beth Bello Cubillos

lizbello77@hotmail.com 